\title{
OPTIMUM EXPRESSION FOR COMPUTATION OF THE GRAVITY FIELD OF A POLYHEDRAL BODY WITH LINEARLY INCREASING DENSITY ${ }^{1}$
}

\author{
V. POHÁNKA ${ }^{2}$
}

\section{ABSTRACT}

The formula for the computation of the gravity field of a polyhedral body with density linearly depending on some coordinate is derived and transformed in the optimum form for numerical calculation.

\section{INTRODUCTION}

The optimum expression for the gravity field of a homogeneous polyhedral body was derived in Pohánka (1988) (hereafter denoted as HPB). However, in many cases the (difference) density within the body varies (in a more or less simple way) with the position. The most usual case is the linear dependence on the depth; therefore, it is desirable to derive an exact formula for the gravity field of a polyhedral body with density linearly depending on some coordinate.

\section{DeRivation OF The Formula}

We follow here the derivation of the formula for the gravity field presented in HPB. If $D$ denotes the interior of the polyhedral body and $\rho(\boldsymbol{r})$ is the (difference) density within the body, then the gravity potential of the body at the point $\boldsymbol{r}$ is

$$
V(\boldsymbol{r})=-\kappa \int_{D} \mathrm{~d} \tau^{\prime} \frac{\rho\left(\boldsymbol{r}^{\prime}\right)}{\left|\boldsymbol{r}^{\prime}-\boldsymbol{r}\right|}
$$

where $\kappa$ is the gravitational constant and $\mathrm{d} \tau^{\prime}$ is the volume element at the point $\boldsymbol{r}^{\prime}$. The intensity of the gravity field is

$$
\begin{aligned}
\boldsymbol{E}(\boldsymbol{r}) & =-\nabla_{\boldsymbol{r}} V(\boldsymbol{r})=\kappa \int_{D} \mathrm{~d} \tau^{\prime} \nabla_{\boldsymbol{r}} \frac{\rho\left(\boldsymbol{r}^{\prime}\right)}{\left|\boldsymbol{r}^{\prime}-\boldsymbol{r}\right|}=-\kappa \int_{D} \mathrm{~d} \tau^{\prime} \rho\left(\boldsymbol{r}^{\prime}\right) \nabla_{\boldsymbol{r}^{\prime}} \frac{1}{\left|\boldsymbol{r}^{\prime}-\boldsymbol{r}\right|}= \\
& =-\kappa \int_{D} \mathrm{~d} \tau^{\prime} \nabla_{\boldsymbol{r}^{\prime}} \frac{\rho\left(\boldsymbol{r}^{\prime}\right)}{\left|\boldsymbol{r}^{\prime}-\boldsymbol{r}\right|}+\kappa \int_{D} \mathrm{~d} \tau^{\prime} \frac{1}{\left|\boldsymbol{r}^{\prime}-\boldsymbol{r}\right|} \nabla_{\boldsymbol{r}^{\prime}} \rho\left(\boldsymbol{r}^{\prime}\right) .
\end{aligned}
$$

In our case the density can be expressed as

$$
\rho(\boldsymbol{r})=\rho_{0}+\boldsymbol{\rho}_{1} \cdot \boldsymbol{r}
$$

(thus $\rho_{0}$ is the value of density in a suitably chosen origin of coordinate system and $\boldsymbol{\rho}_{1}$ is the gradient of density) and we have

$$
\boldsymbol{E}(\boldsymbol{r})=-\kappa \int_{D} \mathrm{~d} \tau^{\prime} \nabla_{\boldsymbol{r}^{\prime}} \frac{\rho_{0}+\boldsymbol{\rho}_{1} \cdot \boldsymbol{r}^{\prime}}{\left|\boldsymbol{r}^{\prime}-\boldsymbol{r}\right|}+\kappa \int_{D} \mathrm{~d} \tau^{\prime} \frac{\boldsymbol{\rho}_{1}}{\left|\boldsymbol{r}^{\prime}-\boldsymbol{r}\right|} .
$$

\footnotetext{
${ }^{1}$ Received January 1997.

${ }^{2}$ Slovak Academy of Sciences, Geophysical Institute, 84228 Bratislava, Dúbravská cesta 9, Slovak Republic
} 
Similarly as in HPB, we express the integrals on the rhs of (4) as surface integrals (over the surface $S$ of the body) using the the Gauss theorem. If $\boldsymbol{f}\left(\boldsymbol{r}^{\prime}\right)$ is a vector function with integrable gradient in the domain $D$, it holds

$$
\int_{D} \mathrm{~d} \tau^{\prime} \nabla_{\boldsymbol{r}^{\prime}} \cdot \boldsymbol{f}\left(\boldsymbol{r}^{\prime}\right)=\int_{S} \mathrm{~d} \boldsymbol{\sigma}^{\prime} \cdot \boldsymbol{f}\left(\boldsymbol{s}^{\prime}\right)
$$

where $\mathrm{d} \boldsymbol{\sigma}^{\prime}$ is the surface element at the point $\boldsymbol{s}^{\prime}$ on the surface $S$ oriented outwards from domain $D$. Accordingly, if $f\left(\boldsymbol{r}^{\prime}\right)$ is a scalar function with integrable gradient in the domain $D$, it holds

$$
\int_{D} \mathrm{~d} \tau^{\prime} \nabla_{\boldsymbol{r}^{\prime}} f\left(\boldsymbol{r}^{\prime}\right)=\int_{S} \mathrm{~d} \boldsymbol{\sigma}^{\prime} f\left(\boldsymbol{s}^{\prime}\right)
$$

In order to transform the second integral on the rhs of (4), we use the identity

$$
\nabla_{\boldsymbol{r}^{\prime}} \cdot \frac{\boldsymbol{r}^{\prime}-\boldsymbol{r}}{\left|\boldsymbol{r}^{\prime}-\boldsymbol{r}\right|}=\frac{2}{\left|\boldsymbol{r}^{\prime}-\boldsymbol{r}\right|}
$$

thus formula (4) reads

$$
\boldsymbol{E}(\boldsymbol{r})=-\kappa \int_{D} \mathrm{~d} \tau^{\prime} \nabla_{\boldsymbol{r}^{\prime}} \frac{\rho_{0}+\boldsymbol{\rho}_{1} \cdot \boldsymbol{r}^{\prime}}{\left|\boldsymbol{r}^{\prime}-\boldsymbol{r}\right|}+\kappa \int_{D} \mathrm{~d} \tau^{\prime} \nabla_{\boldsymbol{r}^{\prime}} \cdot \frac{\boldsymbol{r}^{\prime}-\boldsymbol{r}}{2\left|\boldsymbol{r}^{\prime}-\boldsymbol{r}\right|} \boldsymbol{\rho}_{1}
$$

and according to (5) and (6) we obtain

$$
\boldsymbol{E}(\boldsymbol{r})=-\kappa \int_{S} \mathrm{~d} \boldsymbol{\sigma}^{\prime} \frac{\rho_{0}+\boldsymbol{\rho}_{1} \cdot \boldsymbol{s}^{\prime}}{\left|\boldsymbol{s}^{\prime}-\boldsymbol{r}\right|}+\kappa \int_{S} \mathrm{~d} \boldsymbol{\sigma}^{\prime} \cdot \frac{\boldsymbol{s}^{\prime}-\boldsymbol{r}}{2\left|\boldsymbol{s}^{\prime}-\boldsymbol{r}\right|} \boldsymbol{\rho}_{1} .
$$

Now we write the integrals on the rhs of (8) as a sum of integrals over the sides $S_{k}$ of the polyhedron $(1 \leq k \leq K)$. At any point of the $k$-th side we have $\mathrm{d} \boldsymbol{\sigma}^{\prime}=\boldsymbol{n}_{k} \mathrm{~d} \sigma^{\prime}$, where $\boldsymbol{n}_{k}$ is the unit normal vector pointing outwards from this side and $\mathrm{d} \sigma^{\prime}$ is the scalar surface element; thus

$$
\begin{aligned}
\boldsymbol{E}(\boldsymbol{r}) & =-\kappa \sum_{k=1}^{K} \int_{S_{k}} \mathrm{~d} \sigma^{\prime} \boldsymbol{n}_{k} \frac{\rho_{0}+\boldsymbol{\rho}_{1} \cdot \boldsymbol{s}^{\prime}}{\left|\boldsymbol{s}^{\prime}-\boldsymbol{r}\right|}+\kappa \sum_{k=1}^{K} \int_{S_{k}} \mathrm{~d} \sigma^{\prime} \boldsymbol{n}_{k} \cdot \frac{\boldsymbol{s}^{\prime}-\boldsymbol{r}}{2\left|\boldsymbol{s}^{\prime}-\boldsymbol{r}\right|} \boldsymbol{\rho}_{1}= \\
& =-\kappa \sum_{k=1}^{K} \int_{S_{k}} \mathrm{~d} \sigma^{\prime}\left[\boldsymbol{n}_{k}\left(\frac{\rho_{0}+\boldsymbol{\rho}_{1} \cdot \boldsymbol{r}}{\left|\boldsymbol{s}^{\prime}-\boldsymbol{r}\right|}+\frac{\boldsymbol{\rho}_{1} \cdot\left(\boldsymbol{s}^{\prime}-\boldsymbol{r}\right)}{\left|\boldsymbol{s}^{\prime}-\boldsymbol{r}\right|}\right)-\boldsymbol{n}_{k} \cdot \frac{\boldsymbol{s}^{\prime}-\boldsymbol{r}}{2\left|\boldsymbol{s}^{\prime}-\boldsymbol{r}\right|} \boldsymbol{\rho}_{1}\right]= \\
& =-\kappa \sum_{k=1}^{K}\left[\boldsymbol{n}_{k}\left(\left(\rho_{0}+\boldsymbol{\rho}_{1} \cdot \boldsymbol{r}\right) F_{k}(\boldsymbol{r})+\boldsymbol{\rho}_{1} \cdot \boldsymbol{G}_{k}(\boldsymbol{r})\right)-\frac{1}{2} \boldsymbol{n}_{k} \cdot \boldsymbol{G}_{k}(\boldsymbol{r}) \boldsymbol{\rho}_{1}\right],
\end{aligned}
$$

where

$$
\begin{aligned}
& F_{k}(\boldsymbol{r})=\int_{S_{k}} \mathrm{~d} \sigma^{\prime} \frac{1}{\left|\boldsymbol{s}^{\prime}-\boldsymbol{r}\right|}, \\
& \boldsymbol{G}_{k}(\boldsymbol{r})=\int_{S_{k}} \mathrm{~d} \sigma^{\prime} \frac{\boldsymbol{s}^{\prime}-\boldsymbol{r}}{\left|\boldsymbol{s}^{\prime}-\boldsymbol{r}\right|} .
\end{aligned}
$$

Similarly as in HPB, we express surface integrals in functions $F_{k}(\boldsymbol{r})$ and $\boldsymbol{G}_{k}(\boldsymbol{r})$ as line integrals along the closed curve $L_{k}$, which is the boundary of the side $S_{k}$. This can be done using the 2D Gauss theorem which we express in the following form: if $\boldsymbol{f}\left(\boldsymbol{r}^{\prime}\right)$ is a vector function with integrable gradient in the domain $S_{k}$ (i.e. for $\boldsymbol{r}^{\prime}=\boldsymbol{s}^{\prime}$ ) and $\boldsymbol{n}_{k} \cdot \boldsymbol{f}\left(\boldsymbol{s}^{\prime}\right)=0$ (i.e. vector $\boldsymbol{f}\left(\boldsymbol{s}^{\prime}\right)$ lies in the plane of the side $S_{k}$ ), it holds

$$
\int_{S_{k}} \mathrm{~d} \sigma^{\prime} \nabla_{\boldsymbol{s}^{\prime}} \cdot \boldsymbol{f}\left(\boldsymbol{s}^{\prime}\right)=\int_{L_{k}} \mathrm{~d} \boldsymbol{\xi}^{\prime} \cdot \boldsymbol{f}\left(\boldsymbol{l}^{\prime}\right)
$$


where $\mathrm{d} \boldsymbol{\xi}^{\prime}$ is the linear element at the point $\boldsymbol{l}^{\prime}$ of the curve $L_{k}$ (vector $\mathrm{d} \boldsymbol{\xi}^{\prime}$ is orthogonal to the curve $L_{k}$ and vector $\boldsymbol{n}_{k}$, and it is oriented outwards from the domain $S_{k}$ ).

For correctness we note that we do not (explicitly) use an operator of the 2D gradient: operator $\nabla_{\boldsymbol{r}^{\prime}}$ represents always the $3 \mathrm{D}$ gradient and expression $\nabla_{\boldsymbol{s}^{\prime}} \cdot \boldsymbol{f}\left(\boldsymbol{s}^{\prime}\right)$ is in fact a limit of $\nabla_{\boldsymbol{r}^{\prime}} \cdot \boldsymbol{f}\left(\boldsymbol{r}^{\prime}\right)$ for $\boldsymbol{r}^{\prime} \rightarrow \boldsymbol{s}^{\prime}$. Nevertheless, this expression does not contain the derivative with respect to coordinate perpendicular to the side $S_{k}$ (thus it is in fact a $2 \mathrm{D}$ divergence), as $\boldsymbol{n}_{k} \cdot \boldsymbol{f}\left(\boldsymbol{s}^{\prime}\right)=0$ (here it is important that $\boldsymbol{n}_{k}$ is a constant vector).

In the case that $\boldsymbol{f}\left(\boldsymbol{r}^{\prime}\right)=\boldsymbol{g}\left(\boldsymbol{r}^{\prime}\right) \cdot \boldsymbol{v}$, where $\boldsymbol{v}$ is a constant vector, $\boldsymbol{g}\left(\boldsymbol{r}^{\prime}\right)$ is a tensor function with integrable gradient in the domain $S_{k}$ and $\boldsymbol{n}_{k} \cdot \boldsymbol{g}\left(\boldsymbol{s}^{\prime}\right)=\mathbf{0}$ (i.e. vector $\boldsymbol{g}\left(\boldsymbol{s}^{\prime}\right) \cdot \boldsymbol{v}$ lies in the plane of the side $S_{k}$ ), we get from $(12)$

$$
\left(\int_{S_{k}} \mathrm{~d} \sigma^{\prime} \nabla_{\boldsymbol{s}^{\prime}} \cdot \boldsymbol{g}\left(\boldsymbol{s}^{\prime}\right)\right) \cdot \boldsymbol{v}=\int_{S_{k}} \mathrm{~d} \sigma^{\prime} \nabla_{\boldsymbol{s}^{\prime}} \cdot \boldsymbol{g}\left(\boldsymbol{s}^{\prime}\right) \cdot \boldsymbol{v}=\int_{L_{k}} \mathrm{~d} \boldsymbol{\xi}^{\prime} \cdot \boldsymbol{g}\left(\boldsymbol{l}^{\prime}\right) \cdot \boldsymbol{v}=\left(\int_{L_{k}} \mathrm{~d} \boldsymbol{\xi}^{\prime} \cdot \boldsymbol{g}\left(\boldsymbol{l}^{\prime}\right)\right) \cdot \boldsymbol{v} .
$$

As this holds for any (constant) vector $\boldsymbol{v}$, we obtain the formula

$$
\int_{S_{k}} \mathrm{~d} \sigma^{\prime} \nabla_{\boldsymbol{s}^{\prime}} \cdot \boldsymbol{g}\left(\boldsymbol{s}^{\prime}\right)=\int_{L_{k}} \mathrm{~d} \boldsymbol{\xi}^{\prime} \cdot \boldsymbol{g}\left(\boldsymbol{l}^{\prime}\right)
$$

(note that it is not necessary that tensor $\boldsymbol{g}\left(\boldsymbol{s}^{\prime}\right)$ is a 2D tensor, thus it need not be $\boldsymbol{g}\left(\boldsymbol{s}^{\prime}\right) \cdot \boldsymbol{n}_{k}=\mathbf{0}$ ). For explanation, if $\boldsymbol{u}, \boldsymbol{v}$ are vectors and $\boldsymbol{g}$ is a tensor, $\boldsymbol{u} \cdot \boldsymbol{g}$ and $\boldsymbol{g} \boldsymbol{u}$ are vectors obtained by the scalar multiplication of tensor $\boldsymbol{g}$ with vector $\boldsymbol{u}$ from the left and right, respectively, and $\boldsymbol{u} \cdot \boldsymbol{g} \cdot \boldsymbol{v}$ is a scalar obtained by the scalar multiplication of tensor $\boldsymbol{g}$ with vector $\boldsymbol{u}$ from the left and vector $\boldsymbol{v}$ from the right (the order of multiplications is not substantial).

For the function $F_{k}(\boldsymbol{r})$ it was shown in this way in HPB that

$$
F_{k}(\boldsymbol{r})=\int_{L_{k}} \mathrm{~d} \boldsymbol{\xi}^{\prime} \cdot \frac{\boldsymbol{l}^{\prime}-\boldsymbol{r}}{\left|\boldsymbol{l}^{\prime}-\boldsymbol{r}\right|+\left|\boldsymbol{n}_{k} \cdot\left(\boldsymbol{l}^{\prime}-\boldsymbol{r}\right)\right|} ;
$$

thus we need to transform only the surface integral in $\boldsymbol{G}_{k}(\boldsymbol{r})$. We are looking for a tensor function $\boldsymbol{g}_{k}\left(\boldsymbol{r}^{\prime}\right)$ such that

$$
\nabla_{\boldsymbol{r}^{\prime}} \cdot \boldsymbol{g}_{k}\left(\boldsymbol{r}^{\prime}\right)=\frac{\boldsymbol{r}^{\prime}-\boldsymbol{r}}{\left|\boldsymbol{r}^{\prime}-\boldsymbol{r}\right|}, \quad \boldsymbol{n}_{k} \cdot \boldsymbol{g}_{k}\left(\boldsymbol{r}^{\prime}\right)=\mathbf{0}
$$

Note that it is necessary that these conditions hold only in the domain $S_{k}$; however, for simplicity we require that they hold in the whole space (as this does not represent any loss of generality). We construct tensor $\boldsymbol{g}_{k}\left(\boldsymbol{r}^{\prime}\right)$ from the unit tensor $\boldsymbol{I}$ and vectors $\boldsymbol{r}^{\prime}-\boldsymbol{r}$ and $\boldsymbol{n}_{k}$ using the scalar and tensor product of vectors. We denote the tensor product of vectors $\boldsymbol{u}$ and $\boldsymbol{v}$ (in this order) simply as $\boldsymbol{u} \boldsymbol{v}$ (this tensor is a dyad composed from vectors $\boldsymbol{u}$ and $\boldsymbol{v}$ ).

We first decompose vector $\boldsymbol{r}^{\prime}-\boldsymbol{r}$ into two components, $\left(\boldsymbol{r}^{\prime}-\boldsymbol{r}\right)_{\|}$which is parallel to the side $S_{k}$ and $\left(\boldsymbol{r}^{\prime}-\boldsymbol{r}\right)_{\perp}$ perpendicular to the side $S_{k}$,

$$
\begin{aligned}
& \left(\boldsymbol{r}^{\prime}-\boldsymbol{r}\right)_{\perp}=Z_{k} \boldsymbol{n}_{k}, \quad Z_{k}=\boldsymbol{n}_{k} \cdot\left(\boldsymbol{r}^{\prime}-\boldsymbol{r}\right), \\
& \left(\boldsymbol{r}^{\prime}-\boldsymbol{r}\right)_{\|}=\boldsymbol{r}^{\prime}-\boldsymbol{r}-\left(\boldsymbol{r}^{\prime}-\boldsymbol{r}\right)_{\perp}
\end{aligned}
$$

and denote

$$
\rho_{k}=\left|\left(\boldsymbol{r}^{\prime}-\boldsymbol{r}\right)_{\|}\right|, \quad z_{k}=\left|Z_{k}\right| .
$$

Then the most general form of tensor $\boldsymbol{g}_{k}\left(\boldsymbol{r}^{\prime}\right)$ constructed as mentioned above and satisfying the second condition of (15) is

$$
\boldsymbol{g}_{k}\left(\boldsymbol{r}^{\prime}\right)=\left(\boldsymbol{I}-\boldsymbol{n}_{k} \boldsymbol{n}_{k}\right) g_{0}\left(\rho_{k}, Z_{k}\right)+\left(\boldsymbol{r}^{\prime}-\boldsymbol{r}\right)_{\|}\left(\left(\boldsymbol{r}^{\prime}-\boldsymbol{r}\right)_{\|} g_{1}\left(\rho_{k}, Z_{k}\right)+\boldsymbol{n}_{k} g_{2}\left(\rho_{k}, Z_{k}\right)\right)
$$


We have

$$
\begin{aligned}
& \nabla_{\boldsymbol{r}^{\prime}}\left(\boldsymbol{r}^{\prime}-\boldsymbol{r}\right)_{\|}=\boldsymbol{I}-\boldsymbol{n}_{k} \boldsymbol{n}_{k}, \quad \nabla_{\boldsymbol{r}^{\prime}} \cdot\left(\boldsymbol{r}^{\prime}-\boldsymbol{r}\right)_{\|}=2, \\
& \nabla_{\boldsymbol{r}^{\prime}} g_{i}\left(\rho_{k}, Z_{k}\right)=\frac{\left(\boldsymbol{r}^{\prime}-\boldsymbol{r}\right)_{\|}}{\rho_{k}} \frac{\partial}{\partial \rho_{k}} g_{i}\left(\rho_{k}, Z_{k}\right)+\boldsymbol{n}_{k} \frac{\partial}{\partial Z_{k}} g_{i}\left(\rho_{k}, Z_{k}\right), \\
& \left(\boldsymbol{r}^{\prime}-\boldsymbol{r}\right)_{\|} \cdot \nabla_{\boldsymbol{r}^{\prime}} g_{i}\left(\rho_{k}, Z_{k}\right)=\rho_{k} \frac{\partial}{\partial \rho_{k}} g_{i}\left(\rho_{k}, Z_{k}\right),
\end{aligned}
$$

and thus

$$
\begin{aligned}
& \nabla_{\boldsymbol{r}^{\prime}} \cdot \boldsymbol{g}_{k}\left(\boldsymbol{r}^{\prime}\right)=\left(\boldsymbol{I}-\boldsymbol{n}_{k} \boldsymbol{n}_{k}\right) \cdot \nabla_{\boldsymbol{r}^{\prime}} g_{0}\left(\rho_{k}, Z_{k}\right)+ \\
& +\left(\nabla_{\boldsymbol{r}^{\prime}} \cdot\left(\boldsymbol{r}^{\prime}-\boldsymbol{r}\right)_{\|}\right)\left(\left(\boldsymbol{r}^{\prime}-\boldsymbol{r}\right)_{\|} g_{1}\left(\rho_{k}, Z_{k}\right)+\boldsymbol{n}_{k} g_{2}\left(\rho_{k}, Z_{k}\right)\right)+ \\
& +\left(\boldsymbol{r}^{\prime}-\boldsymbol{r}\right)_{\|} \cdot\left(\left(\nabla_{\boldsymbol{r}^{\prime}}\left(\boldsymbol{r}^{\prime}-\boldsymbol{r}\right)_{\|}\right) g_{1}\left(\rho_{k}, Z_{k}\right)+\right. \\
& \left.+\left(\nabla_{\boldsymbol{r}^{\prime}} g_{1}\left(\rho_{k}, Z_{k}\right)\right)\left(\boldsymbol{r}^{\prime}-\boldsymbol{r}\right)_{\|}+\left(\nabla_{\boldsymbol{r}^{\prime}} g_{2}\left(\rho_{k}, Z_{k}\right)\right) \boldsymbol{n}_{k}\right)= \\
& =\frac{\left(\boldsymbol{r}^{\prime}-\boldsymbol{r}\right)_{\|}}{\rho_{k}} \frac{\partial}{\partial \rho_{k}} g_{0}\left(\rho_{k}, Z_{k}\right)+2\left(\left(\boldsymbol{r}^{\prime}-\boldsymbol{r}\right)_{\|} g_{1}\left(\rho_{k}, Z_{k}\right)+\boldsymbol{n}_{k} g_{2}\left(\rho_{k}, Z_{k}\right)\right)+ \\
& +\left(\left(\boldsymbol{r}^{\prime}-\boldsymbol{r}\right)_{\|} g_{1}\left(\rho_{k}, Z_{k}\right)+\left(\boldsymbol{r}^{\prime}-\boldsymbol{r}\right)_{\|} \rho_{k} \frac{\partial}{\partial \rho_{k}} g_{1}\left(\rho_{k}, Z_{k}\right)+\boldsymbol{n}_{k} \rho_{k} \frac{\partial}{\partial \rho_{k}} g_{2}\left(\rho_{k}, Z_{k}\right)\right)= \\
& =\left(\boldsymbol{r}^{\prime}-\boldsymbol{r}\right)_{\|}\left(\frac{1}{\rho_{k}} \frac{\partial}{\partial \rho_{k}} g_{0}\left(\rho_{k}, Z_{k}\right)+3 g_{1}\left(\rho_{k}, Z_{k}\right)+\rho_{k} \frac{\partial}{\partial \rho_{k}} g_{1}\left(\rho_{k}, Z_{k}\right)\right)+ \\
& +\boldsymbol{n}_{k}\left(2 g_{2}\left(\rho_{k}, Z_{k}\right)+\rho_{k} \frac{\partial}{\partial \rho_{k}} g_{2}\left(\rho_{k}, Z_{k}\right)\right) .
\end{aligned}
$$

Inserting in the first condition of (15) and using the equality

$$
\frac{\boldsymbol{r}^{\prime}-\boldsymbol{r}}{\left|\boldsymbol{r}^{\prime}-\boldsymbol{r}\right|}=\frac{\left(\boldsymbol{r}^{\prime}-\boldsymbol{r}\right)_{\|}+Z_{k} \boldsymbol{n}_{k}}{\sqrt{\rho_{k}^{2}+z_{k}^{2}}}
$$

we obtain equations

$$
\begin{aligned}
& \frac{1}{\rho_{k}} \frac{\partial}{\partial \rho_{k}} g_{0}\left(\rho_{k}, Z_{k}\right)+3 g_{1}\left(\rho_{k}, Z_{k}\right)+\rho_{k} \frac{\partial}{\partial \rho_{k}} g_{1}\left(\rho_{k}, Z_{k}\right)=\frac{1}{\sqrt{\rho_{k}^{2}+z_{k}^{2}}} \\
& 2 g_{2}\left(\rho_{k}, Z_{k}\right)+\rho_{k} \frac{\partial}{\partial \rho_{k}} g_{2}\left(\rho_{k}, Z_{k}\right)=\frac{Z_{k}}{\sqrt{\rho_{k}^{2}+z_{k}^{2}}} .
\end{aligned}
$$

Thus we have

$$
\begin{aligned}
& \frac{\partial}{\partial \rho} g_{0}(\rho, Z)+\frac{1}{\rho} \frac{\partial}{\partial \rho} \rho^{3} g_{1}(\rho, Z)=\frac{\rho}{\sqrt{\rho^{2}+z^{2}}}, \\
& \frac{\partial}{\partial \rho} \rho^{2} g_{2}(\rho, Z)=Z \frac{\rho}{\sqrt{\rho^{2}+z^{2}}},
\end{aligned}
$$

where $z=|Z|$. In order to use equality (13) we require that tensor $\boldsymbol{g}_{k}\left(\boldsymbol{r}^{\prime}\right)$ is bounded for $\boldsymbol{r}^{\prime} \rightarrow \boldsymbol{r}$; this implies that $g_{0}(\rho, Z), \rho^{2} g_{1}(\rho, Z)$ and $\rho g_{2}(\rho, Z)$ have to be bounded for $\rho \rightarrow 0+$. Then the single solution of $(20)$ is

$$
g_{2}(\rho, Z)=\frac{Z}{\rho^{2}}\left(\sqrt{\rho^{2}+z^{2}}-z\right)=\frac{Z}{\sqrt{\rho^{2}+z^{2}}+z}
$$


(note that it is always $z \geq 0$ ); in the case of (19) we are free to choose one of the functions $g_{0}(\rho, Z), g_{1}(\rho, Z)$.

In view of (13), (15) and (18) we get from (11)

$$
\begin{aligned}
\boldsymbol{G}_{k}(\boldsymbol{r}) & =\int_{S_{k}} \mathrm{~d} \sigma^{\prime} \nabla_{\boldsymbol{s}^{\prime}} \cdot \boldsymbol{g}_{k}\left(\boldsymbol{s}^{\prime}\right)=\int_{L_{k}} \mathrm{~d} \boldsymbol{\xi}^{\prime} \cdot \boldsymbol{g}_{k}\left(\boldsymbol{l}^{\prime}\right)= \\
& =\int_{L_{k}} \mathrm{~d} \boldsymbol{\xi}^{\prime} \cdot\left[\left(\boldsymbol{I}-\boldsymbol{n}_{k} \boldsymbol{n}_{k}\right) g_{0}\left(\rho_{k}, Z_{k}\right)+\left(\boldsymbol{l}^{\prime}-\boldsymbol{r}\right)_{\|}\left(\left(\boldsymbol{l}^{\prime}-\boldsymbol{r}\right)_{\|} g_{1}\left(\rho_{k}, Z_{k}\right)+\boldsymbol{n}_{k} g_{2}\left(\rho_{k}, Z_{k}\right)\right)\right] .
\end{aligned}
$$

In our case $L_{k}$ is a closed broken line composed from $L(k)$ line segments $L_{k, l}(1 \leq l \leq L(k))$; we assume that these segments are numbered in the direct sense (counter-clockwise) viewed from the outside of the body. Vertices of the side $S_{k}$ are numbered in the same order: end points of segment $L_{k, l}$ are denoted as $\boldsymbol{a}_{k, l}$ and $\boldsymbol{a}_{k, l+1}$ (thus $\boldsymbol{a}_{k, L(k)+1}=\boldsymbol{a}_{k, 1}$ ). For every segment $L_{k, l}$ we define two unit vectors: vector $\boldsymbol{\mu}_{k, l}$, which is parallel with the segment and has the same orientation

$$
\boldsymbol{\mu}_{k, l}=\frac{\boldsymbol{a}_{k, l+1}-\boldsymbol{a}_{k, l}}{d_{k, l}}
$$

where $d_{k, l}$ is the length of the segment $L_{k, l}$

$$
d_{k, l}=\left|\boldsymbol{a}_{k, l+1}-\boldsymbol{a}_{k, l}\right|,
$$

and vector $\boldsymbol{\nu}_{k, l}$ lying in the plane of the side $S_{k}$, perpendicular to the segment $L_{k, l}$ and directed outwards from the side $S_{k}$, which reads

$$
\boldsymbol{\nu}_{k, l}=\boldsymbol{\mu}_{k, l} \times \boldsymbol{n}_{k} .
$$

Then we have on the segment $L_{k, l}$

$$
\boldsymbol{l}^{\prime}=\boldsymbol{a}_{k, l}+\boldsymbol{\mu}_{k, l} \xi^{\prime} \quad\left(0 \leq \xi^{\prime} \leq d_{k, l}\right)
$$

and

$$
\mathrm{d} \boldsymbol{\xi}^{\prime}=\boldsymbol{\nu}_{k, l} \mathrm{~d} \xi^{\prime}
$$

Further we denote

$$
\begin{aligned}
& u_{k, l}(\boldsymbol{r})=\boldsymbol{\mu}_{k, l} \cdot\left(\boldsymbol{a}_{k, l}-\boldsymbol{r}\right), \\
& v_{k, l}(\boldsymbol{r})=u_{k, l}(\boldsymbol{r})+d_{k, l}, \\
& w_{k, l}(\boldsymbol{r})=\boldsymbol{\nu}_{k, l} \cdot\left(\boldsymbol{a}_{k, l}-\boldsymbol{r}\right) ;
\end{aligned}
$$

and

$$
Z_{k}(\boldsymbol{r})=\boldsymbol{n}_{k} \cdot\left(\boldsymbol{a}_{k, 1}-\boldsymbol{r}\right), \quad z_{k}(\boldsymbol{r})=\left|Z_{k}(\boldsymbol{r})\right|,
$$

As the quantity $\boldsymbol{n}_{k} \cdot\left(\boldsymbol{l}^{\prime}-\boldsymbol{r}\right)\left(=\boldsymbol{n}_{k} \cdot\left(\boldsymbol{a}_{k, l}-\boldsymbol{r}\right)\right)$ does not depend on $\xi^{\prime}$ nor on the index $l$, this quantity is equal to $Z_{k}(\boldsymbol{r})$ (on any segment of $S_{k}$ ); thus we have according to (16) and (17) on the segment $L_{k, l}$

$$
\begin{aligned}
& Z_{k}=Z_{k}(\boldsymbol{r}), \quad z_{k}=z_{k}(\boldsymbol{r}), \\
& \left(\boldsymbol{l}^{\prime}-\boldsymbol{r}\right)_{\|}=\left(u_{k, l}(\boldsymbol{r})+\xi^{\prime}\right) \boldsymbol{\mu}_{k, l}+w_{k, l}(\boldsymbol{r}) \boldsymbol{\nu}_{k, l}, \\
& \rho_{k}=\rho\left(u_{k, l}(\boldsymbol{r})+\xi^{\prime}, w_{k, l}(\boldsymbol{r})\right), \quad \rho(\xi, w)=\sqrt{\xi^{2}+w^{2}} .
\end{aligned}
$$


Now we can write (22) in the form

$$
\begin{gathered}
\boldsymbol{G}_{k}(\boldsymbol{r})=\sum_{l=1}^{L(k)} \int_{L_{k, l}} \mathrm{~d} \boldsymbol{\xi}^{\prime} \cdot\left[\left(\boldsymbol{I}-\boldsymbol{n}_{k} \boldsymbol{n}_{k}\right) g_{0}\left(\rho_{k}, Z_{k}\right)+\left(\boldsymbol{l}^{\prime}-\boldsymbol{r}\right)_{\|}\left(\left(\boldsymbol{l}^{\prime}-\boldsymbol{r}\right)_{\|} g_{1}\left(\rho_{k}, Z_{k}\right)+\boldsymbol{n}_{k} g_{2}\left(\rho_{k}, Z_{k}\right)\right)\right]= \\
=\sum_{l=1}^{L(k)} \int_{0}^{d_{k, l}} \mathrm{~d} \xi^{\prime}\left[\boldsymbol{\nu}_{k, l} g_{0}\left(\rho_{k}, Z_{k}\right)+\boldsymbol{\nu}_{k, l} \cdot\left(\boldsymbol{l}^{\prime}-\boldsymbol{r}\right)_{\|}\left(\left(\boldsymbol{l}^{\prime}-\boldsymbol{r}\right)_{\|} g_{1}\left(\rho_{k}, Z_{k}\right)+\boldsymbol{n}_{k} g_{2}\left(\rho_{k}, Z_{k}\right)\right)\right]= \\
=\sum_{l=1}^{L(k)} \int_{0}^{d_{k, l}} \mathrm{~d} \xi^{\prime}\left[\boldsymbol{\nu}_{k, l} g_{0}\left(\rho\left(u_{k, l}(\boldsymbol{r})+\xi^{\prime}, w_{k, l}(\boldsymbol{r})\right), Z_{k}(\boldsymbol{r})\right)+\right. \\
+w_{k, l}(\boldsymbol{r})\left(\left(\left(u_{k, l}(\boldsymbol{r})+\xi^{\prime}\right) \boldsymbol{\mu}_{k, l}+w_{k, l}(\boldsymbol{r}) \boldsymbol{\nu}_{k, l}\right) g_{1}\left(\rho\left(u_{k, l}(\boldsymbol{r})+\xi^{\prime}, w_{k, l}(\boldsymbol{r})\right), Z_{k}(\boldsymbol{r})\right)+\right. \\
\left.\left.\quad+\boldsymbol{n}_{k} g_{2}\left(\rho\left(u_{k, l}(\boldsymbol{r})+\xi^{\prime}, w_{k, l}(\boldsymbol{r})\right), Z_{k}(\boldsymbol{r})\right)\right)\right]= \\
\begin{array}{c}
\sum_{l=1}^{L(k)} \int_{u_{k, l}(\boldsymbol{r})}^{v_{k, l}(\boldsymbol{r})} \mathrm{d} \xi\left[\boldsymbol{\nu}_{k, l} g_{0}\left(\rho\left(\xi, w_{k, l}(\boldsymbol{r})\right), Z_{k}(\boldsymbol{r})\right)+\right. \\
+w_{k, l}(\boldsymbol{r})\left(\left(\xi \boldsymbol{\mu}_{k, l}+w_{k, l}(\boldsymbol{r}) \boldsymbol{\nu}_{k, l}\right) g_{1}\left(\rho\left(\xi, w_{k, l}(\boldsymbol{r})\right), Z_{k}(\boldsymbol{r})\right)+\right. \\
\left.\left.+\boldsymbol{n}_{k} g_{2}\left(\rho\left(\xi, w_{k, l}(\boldsymbol{r})\right), Z_{k}(\boldsymbol{r})\right)\right)\right] .
\end{array}
\end{gathered}
$$

If we denote

$$
\begin{aligned}
& \Phi_{1}(u, v, w, Z)=\int_{u}^{v} \mathrm{~d} \xi w \xi g_{1}(\rho(\xi, w), Z), \\
& \Phi_{2}(u, v, w, Z)=\int_{u}^{v} \mathrm{~d} \xi\left(g_{0}(\rho(\xi, w), Z)+w^{2} g_{1}(\rho(\xi, w), Z)\right), \\
& \Phi_{3}(u, v, w, Z)=\int_{u}^{v} \mathrm{~d} \xi w g_{2}(\rho(\xi, w), Z),
\end{aligned}
$$

we obtain

$$
\begin{aligned}
\boldsymbol{G}_{k}(\boldsymbol{r})=\sum_{l=1}^{L(k)}[ & \Phi_{1}\left(u_{k, l}(\boldsymbol{r}), v_{k, l}(\boldsymbol{r}), w_{k, l}(\boldsymbol{r}), Z_{k}(\boldsymbol{r})\right) \boldsymbol{\mu}_{k, l}+\Phi_{2}\left(u_{k, l}(\boldsymbol{r}), v_{k, l}(\boldsymbol{r}), w_{k, l}(\boldsymbol{r}), Z_{k}(\boldsymbol{r})\right) \boldsymbol{\nu}_{k, l}+ \\
+ & \left.\Phi_{3}\left(u_{k, l}(\boldsymbol{r}), v_{k, l}(\boldsymbol{r}), w_{k, l}(\boldsymbol{r}), Z_{k}(\boldsymbol{r})\right) \boldsymbol{n}_{k}\right] .
\end{aligned}
$$

Using (21) we get

$$
\Phi_{3}(u, v, w, Z)=Z \Phi(u, v, w, Z)
$$

where

$$
\Phi(u, v, w, Z)=\int_{u}^{v} \mathrm{~d} \xi \frac{w}{\sqrt{\xi^{2}+w^{2}+z^{2}}+z},
$$

$(z=|Z|$ and thus $\Phi(u, v, w, Z)=\Phi(u, v, w, z))$. In HPB this function is denoted as $\phi(u, v, w, z)$ and it was shown that (14) can be expressed as

$$
F_{k}(\boldsymbol{r})=\sum_{l=1}^{L(k)} \Phi\left(u_{k, l}(\boldsymbol{r}), v_{k, l}(\boldsymbol{r}), w_{k, l}(\boldsymbol{r}), z_{k}(\boldsymbol{r})\right) .
$$


Explicit formula for the function $\Phi(u, v, w, z)$ reads

$$
\Phi(u, v, w, z)=w L(u, v, w, z)+2 z A(u, v, w, z)
$$

where

$$
\begin{aligned}
& L(u, v, w, z)=\ln \frac{\sqrt{v^{2}+w^{2}+z^{2}}+v}{\sqrt{u^{2}+w^{2}+z^{2}}+u}, \\
& A(u, v, w, z)=-\arctan \frac{2 w(v-u)}{T(u, v, w, z)^{2}-(v-u)^{2}+2 T(u, v, w, z) z}, \\
& T(u, v, w, z)=\sqrt{u^{2}+w^{2}+z^{2}}+\sqrt{v^{2}+w^{2}+z^{2}} .
\end{aligned}
$$

Integral in the first formula of (32) can be removed if we put

$$
g_{1}(\rho, Z)=\frac{1}{\rho} \frac{\partial}{\partial \rho} h(\rho, Z)
$$

then we obtain using the last formula of (30)

$$
\Phi_{1}(u, v, w, Z)=\int_{u}^{v} \mathrm{~d} \xi w \frac{\partial}{\partial \xi} h(\rho(\xi, w), Z)=w(h(\rho(v, w), Z)-h(\rho(u, w), Z)) .
$$

Inserting (41) in the condition (19) we get

$$
\frac{\partial}{\partial \rho} g_{0}(\rho, Z)+\frac{1}{\rho} \frac{\partial}{\partial \rho} \rho^{2} \frac{\partial}{\partial \rho} h(\rho, Z)=\frac{\rho}{\sqrt{\rho^{2}+z^{2}}},
$$

what can be written in the form

$$
\frac{\partial}{\partial \rho}\left(g_{0}(\rho, Z)+\frac{\partial}{\partial \rho} \rho h(\rho, Z)\right)=\frac{\partial}{\partial \rho} \sqrt{\rho^{2}+z^{2}}
$$

(note that equations $(41)-(43)$ are not changed if we replace $h(\rho, Z)$ by $h(\rho, Z)+c(Z)$, where $c(Z)$ is a function of $Z$ ). Thus we have

$$
g_{0}(\rho, Z)+\frac{\partial}{\partial \rho} \rho h(\rho, Z)=\sqrt{\rho^{2}+z^{2}}
$$

as the additional integration term of the form $c(Z)$ on the rhs can be removed if we replace $h(\rho, Z)$ by $h(\rho, Z)+c(Z)$. Then we have

$$
g_{0}(\rho, Z)+w^{2} g_{1}(\rho, Z)=\sqrt{\rho^{2}+z^{2}}-h(\rho, Z)-\frac{\rho^{2}-w^{2}}{\rho} \frac{\partial}{\partial \rho} h(\rho, Z),
$$

using the last formula of (30) we get

$$
g_{0}(\rho(\xi, w), Z)+w^{2} g_{1}(\rho(\xi, w), Z)=\sqrt{\xi^{2}+w^{2}+z^{2}}-\frac{\partial}{\partial \xi} \xi h(\rho(\xi, w), Z),
$$

and from the second formula of (32) we obtain

$$
\begin{aligned}
\Phi_{2}(u, v, w, Z) & =\int_{u}^{v} \mathrm{~d} \xi\left(\sqrt{\xi^{2}+w^{2}+z^{2}}-\frac{\partial}{\partial \xi} \xi h(\rho(\xi, w), Z)\right)= \\
& =\int_{u}^{v} \mathrm{~d} \xi \sqrt{\xi^{2}+w^{2}+z^{2}}-(v h(\rho(v, w), Z)-u h(\rho(u, w), Z)) .
\end{aligned}
$$


We can easily calculate the indefinite integral

$$
\int \mathrm{d} \xi \sqrt{\xi^{2}+w^{2}+z^{2}}=\frac{1}{2}\left(\xi \sqrt{\xi^{2}+w^{2}+z^{2}}+\left(w^{2}+z^{2}\right) \ln \left(\sqrt{\xi^{2}+w^{2}+z^{2}}+\xi\right)\right)
$$

thus the integral on the rhs of $(45)$ is

$$
\int_{u}^{v} \mathrm{~d} \xi \sqrt{\xi^{2}+w^{2}+z^{2}}=\frac{1}{2}\left(v \sqrt{v^{2}+w^{2}+z^{2}}-u \sqrt{u^{2}+w^{2}+z^{2}}+\left(w^{2}+z^{2}\right) L(u, v, w, z)\right)
$$

and we finally have

$$
\begin{aligned}
\Phi_{2}(u, v, w, Z)= & \frac{1}{2}\left(v \sqrt{v^{2}+w^{2}+z^{2}}-u \sqrt{u^{2}+w^{2}+z^{2}}+\left(w^{2}+z^{2}\right) L(u, v, w, z)\right)- \\
& -(v h(\rho(v, w), Z)-u h(\rho(u, w), Z)) .
\end{aligned}
$$

Thus we have expressed functions $\Phi_{1}(u, v, w, Z)$ and $\Phi_{2}(u, v, w, Z)$ (formulae (42) and (47)) in terms of function $h(\rho, Z)$, which has to satisfy only the condition that $h(\rho, Z)$ and $\rho \partial h(\rho, Z) / \partial \rho$ have to be bounded for $\rho \rightarrow 0+$.

Now we are able to determine the form of function $h(\rho, Z)$ in such a way that the expression (33) for the function $\boldsymbol{G}_{k}(\boldsymbol{r})$ would be the simplest possible. We first put

$$
h(\rho, Z)=0
$$

and from (42) and (47) we obtain

$$
\begin{aligned}
& \Phi_{1}(u, v, w, Z)=0 \\
& \Phi_{2}(u, v, w, Z)=\frac{1}{2}\left(v \sqrt{v^{2}+w^{2}+z^{2}}-u \sqrt{u^{2}+w^{2}+z^{2}}+\left(w^{2}+z^{2}\right) L(u, v, w, z)\right) .
\end{aligned}
$$

Now we can try to find still simpler expression for these functions. It can be easily shown that the term containing function $L(u, v, w, z)$ cannot be removed from the expression of function $\Phi_{2}(u, v, w, Z)$ by any choice of function $h(\rho, Z)$ : if it would be for some function $f(\rho, Z)$

$$
v f(\rho(v, w), Z)-u f(\rho(u, w), Z)=\frac{1}{2}\left(w^{2}+z^{2}\right) L(u, v, w, z)
$$

then inserting $u=-v$ we would get

$$
f(\rho(v, w), Z)=\frac{w^{2}+z^{2}}{4 v} \ln \frac{\sqrt{v^{2}+w^{2}+z^{2}}+v}{\sqrt{v^{2}+w^{2}+z^{2}}-v} ;
$$

however, neither the argument of logarithm nor the term in front of it can be expressed as a function of $\rho(v, w)$ and $Z$. This means that function $\Phi_{2}(u, v, w, Z)$ has to contain the logarithm term and that it is not reasonable to consider any function $h(\rho, Z)$ containing any logarithmic or other transcendental function. We can still try to remove or simplify the first term on the rhs of (47): if we put

$$
h(\rho, Z)=\frac{1}{2} \rho(\rho, z),
$$

we obtain

$$
\begin{aligned}
& \Phi_{1}(u, v, w, Z)=\frac{1}{2} w\left(\sqrt{v^{2}+w^{2}+z^{2}}-\sqrt{u^{2}+w^{2}+z^{2}}\right), \\
& \Phi_{2}(u, v, w, Z)=\frac{1}{2}\left(w^{2}+z^{2}\right) L(u, v, w, z) .
\end{aligned}
$$


It is clear that there are no simpler expressions for the functions $\Phi_{1}(u, v, w, Z)$ and $\Phi_{2}(u, v, w, Z)$ than (49) or (51) (note also that in both cases $\Phi_{1}(u, v, w, Z)=\Phi_{1}(u, v, w, z)$ and $\Phi_{2}(u, v, w, Z)=\Phi_{2}(u, v, w, z)$ ).

It has to be noted that the expression of function $\boldsymbol{G}_{k}(\boldsymbol{r})$ corresponding to formulae (33) and (49) was obtained (in a slightly different way and in context of an other problem) by Ivan (1996) (author is indebted to the anonymous reviewer for this information).

Now we can present the resulting formula for the intensity of the gravity field $\boldsymbol{E}(\boldsymbol{r})$ : from (9), (33), (34) and (36) we get

$$
\begin{aligned}
\boldsymbol{E}(\boldsymbol{r})=-\kappa \sum_{k=1}^{K} \sum_{l=1}^{L(k)}[ & \left(\rho_{0}+\boldsymbol{\rho}_{1} \cdot \boldsymbol{r}+\boldsymbol{\rho}_{1} \cdot \boldsymbol{n}_{k} Z_{k}(\boldsymbol{r})\right) \Phi\left(u_{k, l}(\boldsymbol{r}), v_{k, l}(\boldsymbol{r}), w_{k, l}(\boldsymbol{r}), z_{k}(\boldsymbol{r})\right)+ \\
& +\boldsymbol{\rho}_{1} \cdot \boldsymbol{\mu}_{k, l} \Phi_{1}\left(u_{k, l}(\boldsymbol{r}), v_{k, l}(\boldsymbol{r}), w_{k, l}(\boldsymbol{r}), z_{k}(\boldsymbol{r})\right)+ \\
& \left.+\boldsymbol{\rho}_{1} \cdot \boldsymbol{\nu}_{k, l} \Phi_{2}\left(u_{k, l}(\boldsymbol{r}), v_{k, l}(\boldsymbol{r}), w_{k, l}(\boldsymbol{r}), z_{k}(\boldsymbol{r})\right)\right) \boldsymbol{n}_{k}- \\
& \left.-\frac{1}{2} Z_{k}(\boldsymbol{r}) \Phi\left(u_{k, l}(\boldsymbol{r}), v_{k, l}(\boldsymbol{r}), w_{k, l}(\boldsymbol{r}), z_{k}(\boldsymbol{r})\right) \boldsymbol{\rho}_{1}\right]
\end{aligned}
$$

where functions $\Phi(u, v, w, z), \Phi_{1}(u, v, w, z), \Phi_{2}(u, v, w, z)$ are given by (37) and (49) or (51).

\section{Numerical Aspects}

Now we modify the obtained formula for numerical calculation, closely following HPB and Pohánka (1990): we aim to eliminate any undefined operations (e.g. expressions of the type 0/0) and to improve the accuracy of calculation. For the function $\Phi(u, v, w, z)$ this is already done in the mentioned articles: we express function $L(u, v, w, z)$ in the form (note that we have always $u \leq v$ )

$$
\begin{aligned}
& \operatorname{sign}(u)=\operatorname{sign}(v): \quad L(u, v, w, z)=\operatorname{sign}(v) \ln \frac{\sqrt{v^{2}+w^{2}+z^{2}}+|v|}{\sqrt{u^{2}+w^{2}+z^{2}}+|u|}, \\
& \operatorname{sign}(u) \neq \operatorname{sign}(v): \quad L(u, v, w, z)=\ln \frac{\left(\sqrt{v^{2}+w^{2}+z^{2}}+|v|\right)\left(\sqrt{u^{2}+w^{2}+z^{2}}+|u|\right)}{w^{2}+z^{2}},
\end{aligned}
$$

and replace function $\Phi(u, v, w, z)$ (given by (37)) in the formula (52) by the function $\Phi(u, v, w, z, \varepsilon)$ defined as

$$
\Phi(u, v, w, z, \varepsilon)=w L(u, v, w, z+\varepsilon)+2 z A(u, v, w, z+\varepsilon)
$$

where the positive parameter $\varepsilon$ has a dimension of length and it is much smaller than the characteristic dimension of the polyhedron.

In the case of functions $\Phi_{1}(u, v, w, z)$ and $\Phi_{2}(u, v, w, z)$ we first write function $L(u, v, w, z)$ in the form (53) and rewrite the other terms according to equalities

$$
\begin{aligned}
& \sqrt{v^{2}+w^{2}+z^{2}}-\sqrt{u^{2}+w^{2}+z^{2}}=\frac{(v-u)(v+u)}{T(u, v, w, z)} \\
& v \sqrt{v^{2}+w^{2}+z^{2}}-u \sqrt{u^{2}+w^{2}+z^{2}}=\frac{1}{2}(v-u)\left(\frac{(v+u)^{2}}{T(u, v, w, z)}+T(u, v, w, z)\right)
\end{aligned}
$$


in order to decrease the error at points far away from the body. Then we replace functions $\Phi_{1}(u, v, w, z)$, $\Phi_{2}(u, v, w, z)$ in the formula (52) by functions $\Phi_{1}(u, v, w, z, \varepsilon), \Phi_{2}(u, v, w, z, \varepsilon)$, respectively, where

$$
\begin{aligned}
\Phi_{1}(u, v, w, z, \varepsilon)= & 0 \\
\Phi_{2}(u, v, w, z, \varepsilon)= & \frac{1}{4}(v-u)\left(\frac{(v+u)^{2}}{T(u, v, w, z+\varepsilon)}+T(u, v, w, z+\varepsilon)\right)+ \\
& +\frac{1}{2}\left(w^{2}+z^{2}\right) L(u, v, w, z+\varepsilon),
\end{aligned}
$$

in the first case (formula (49)) and

$$
\begin{aligned}
\Phi_{1}(u, v, w, z, \varepsilon) & =\frac{(v-u)(v+u) w}{2 T(u, v, w, z+\varepsilon)}, \\
\Phi_{2}(u, v, w, z, \varepsilon) & =\frac{1}{2}\left(w^{2}+z^{2}\right) L(u, v, w, z+\varepsilon),
\end{aligned}
$$

in the second case (formula (51)). Then the expressions (54), (56) and (57) are well defined for $v \geq u, z \geq 0$ and they are suitable for the numerical calculation.

Finally we compare the two variants (56) and (57) with respect to the speed of calculation. Expression (57) is a little simpler than (56), as it contains two additions less than the other. However, by the calculation of expression (52) using (56) we need neither to calculate the quantity $\boldsymbol{\rho}_{1} \cdot \boldsymbol{\mu}_{k, l}$ (thus perform three multiplications and two additions), nor to multiply it by a nonzero quantity and add to the other terms. Therefore the most simple variant for the numerical calculation is that given by formulae (52), (54) and (56).

\section{Algorithm of Calculation}

Let us repeat briefly the optimum algorithm for the calculation of the gravity field of a polyhedral body with linearly increasing density (we follow here closely the algorithm presented in HPB). The input parameters of the body are:

- value of the density at the origin of coordinates $\rho_{0}$ (note that this origin can lie outside the body),

- gradient of the density $\boldsymbol{\rho}_{1}$ (thus density at the point $\boldsymbol{r}$ within the body is given by $\rho(\boldsymbol{r})=\rho_{0}+\boldsymbol{\rho}_{1} \cdot \boldsymbol{r}$ ),

- number of polyhedron sides $K$,

- for every side (designated by the number $k, 1 \leq k \leq K$ ), the number of edges $L(k)$ (equal to the number of vertices),

- for every vertex of the $k$-th side (designated by the number $l, 1 \leq l \leq L(k)$ ), its radius-vector $\boldsymbol{a}_{k, l}$ (the vertices are ordered in the direct sense viewed from the exterior of the body and $\left.\boldsymbol{a}_{k, L(k)+1}=\boldsymbol{a}_{k, 1}\right)$. The $l$-th edge is defined by its end points $\boldsymbol{a}_{k, l}$ and $\boldsymbol{a}_{k, l+1}$.

The last input parameter is the radius-vector $\boldsymbol{r}$ of the point of calculation of the gravity field.

In the calculation we proceed as follows:

1. For each side (i.e. for each $k$ ) we find the following quantities:

(a) For each edge (i.e. for each $l$ ) its length

$$
d_{k, l}=\left|\boldsymbol{a}_{k, l+1}-\boldsymbol{a}_{k, l}\right|,
$$

and the unit vector

$$
\boldsymbol{\mu}_{k, l}=\frac{\boldsymbol{a}_{k, l+1}-\boldsymbol{a}_{k, l}}{d_{k, l}} .
$$


(b) The unit normal vector $\boldsymbol{n}_{k}$ pointing outwards from the side (see HPB)

$$
\boldsymbol{n}_{k}=\frac{\boldsymbol{N}_{k}}{\left|\boldsymbol{N}_{k}\right|}
$$

where

$$
\boldsymbol{N}_{k}=\sum_{l=2}^{L(k)-1}\left(\boldsymbol{a}_{k, l}-\boldsymbol{a}_{k, 1}\right) \times\left(\boldsymbol{a}_{k, l+1}-\boldsymbol{a}_{k, 1}\right) .
$$

(c) For each edge the unit vector

$$
\boldsymbol{\nu}_{k, l}=\boldsymbol{\mu}_{k, l} \times \boldsymbol{n}_{k} .
$$

None of the quantities mentioned in this paragraph depend on $\boldsymbol{r}$.

2. For the given point $\boldsymbol{r}$ we calculate the following quantities:

(a) For each $k$

$$
Z_{k}=\boldsymbol{n}_{k} \cdot\left(\boldsymbol{a}_{k, 1}-\boldsymbol{r}\right), \quad z_{k}=\left|Z_{k}\right| .
$$

(b) For each $k$ and $l$

$$
\begin{aligned}
& u_{k, l}=\boldsymbol{\mu}_{k, l} \cdot\left(\boldsymbol{a}_{k, l}-\boldsymbol{r}\right), \\
& v_{k, l}=u_{k, l}+d_{k, l}, \\
& w_{k, l}=\boldsymbol{\nu}_{k, l} \cdot\left(\boldsymbol{a}_{k, l}-\boldsymbol{r}\right) .
\end{aligned}
$$

3. For some choice of the small positive parameter $\varepsilon$, the (aproximation of the) intensity of the gravity field $\boldsymbol{E}(\boldsymbol{r}, \varepsilon)$ is given by

$$
\begin{aligned}
\boldsymbol{E}(\boldsymbol{r}, \varepsilon)=-\kappa \sum_{k=1}^{K} \sum_{l=1}^{L(k)}[ & \left(\left(\rho_{0}+\boldsymbol{\rho}_{1} \cdot \boldsymbol{r}+\boldsymbol{\rho}_{1} \cdot \boldsymbol{n}_{k} Z_{k}\right) \Phi\left(u_{k, l}, v_{k, l}, w_{k, l}, z_{k}, \varepsilon\right)+\right. \\
& \left.+\boldsymbol{\rho}_{1} \cdot \boldsymbol{\nu}_{k, l} \Phi_{2}\left(u_{k, l}, v_{k, l}, w_{k, l}, z_{k}, \varepsilon\right)\right) \boldsymbol{n}_{k}- \\
& \left.-\frac{1}{2} Z_{k} \Phi\left(u_{k, l}, v_{k, l}, w_{k, l}, z_{k}, \varepsilon\right) \boldsymbol{\rho}_{1}\right]
\end{aligned}
$$

where functions $\Phi(u, v, w, z, \varepsilon), \Phi_{2}(u, v, w, z, \varepsilon)$ can be calculated as follows. For the given numbers $u, v$ $(v=u+d, d>0), w, z(z \geq 0)$, we make the following consecutive steps:

(a) $z_{\varepsilon}=z+\varepsilon$,

(b) $W^{2}=w^{2}+z^{2}, \quad W_{\varepsilon}^{2}=w^{2}+z_{\varepsilon}^{2}$,

(c) $U_{\varepsilon}=\sqrt{u^{2}+W_{\varepsilon}^{2}}, \quad V_{\varepsilon}=\sqrt{v^{2}+W_{\varepsilon}^{2}}$,

(d) $T_{\varepsilon}=U_{\varepsilon}+V_{\varepsilon}$,

(e) $\operatorname{sign}(u)=\operatorname{sign}(v): \quad L_{\varepsilon}=\operatorname{sign}(v) \ln \frac{V_{\varepsilon}+|v|}{U_{\varepsilon}+|u|}$, 


$$
\begin{aligned}
& \operatorname{sign}(u) \neq \operatorname{sign}(v): \quad L_{\varepsilon}=\ln \frac{\left(V_{\varepsilon}+|v|\right)\left(U_{\varepsilon}+|u|\right)}{W_{\varepsilon}^{2}}, \\
& A_{\varepsilon}=-\arctan \frac{2 w d}{\left(T_{\varepsilon}+d\right)\left|T_{\varepsilon}-d\right|+2 T_{\varepsilon} z_{\varepsilon}}, \\
& \text { (f) } \Phi(u, v, w, z, \varepsilon)=w L_{\varepsilon}+2 z A_{\varepsilon}, \\
& \Phi_{2}(u, v, w, z, \varepsilon)=\frac{1}{4} d\left(\frac{(v+u)^{2}}{T_{\varepsilon}}+T_{\varepsilon}\right)+\frac{1}{2} W^{2} L_{\varepsilon} .
\end{aligned}
$$

\section{CONCLUding REMARKS}

We have derived a formula for the computation of the gravity field of a polyhedral body with linearly increasing density. This formula has the following properties: (1) it is maximally simple, (2) it is valid for every point of space, and (3) it needs no special attention for the points near or on the surface of the body. However, when compared with the formula for a polyhedral body with constant density, there has to be paid an attention for the points very far from the body. This is because of the behaviour of the terms of the sum on the rhs of formula (52): these terms behave for large distances from the body as polynomials of coordinates of second order, compared with polynomials of the first order in the case of constant density. Therefore it is necessary to use by the numerical calculation higher precision expression of real numbers.

Acknowledgement. The author is grateful to the Slovak Grant agency for science (grant No. 2/1063) for the partial support of this work.

\section{REFERENCES}

Ivan, M. 1996. Polyhedral approximations in physical geodesy. Journal of Geodesy 70, 755-767.

PoHÁnkA, V. 1988. Optimum expression for computation of the gravity field of a homogeneous polyhedral body. Geophysical Prospecting 36, 733-751.

PoнÁnka, V. 1990. Reply to comment by M. Ivan. Geophysical Prospecting 38, 333-335. 